\section{Autopercepción de competencias genéricas de egresados de Odontología año 2016-2017, de una universidad privada, en Lima-Perú}

\section{Self-perception of generic competences of graduates of Odontology 2016-2017, of a private university, in Lima-Peru}

\begin{abstract}
Resumen
Objetivo. Evaluar la autopercepción de los egresados de Odontología en cuanto al logro de las competencias genéricas al término de la formación profesional. Métodos. Enfoque cuantitativo, diseńo descriptivo y transversal. Se aplicó una encuesta estructurada conformada por tres dimensiones, dirigida a 137 egresados periodo 2016-2017. Se estableció cuatro niveles de logro de la competencia (N1, N2, N3 y N4). Resultados. El compromiso con el desarrollo personal y profesional; el compromiso y responsabilidad con el entorno social y medio ambiente, así como la demostración de comportamiento y compromiso ético fueron las competencias más reportadas en el nivel 4, con valores $83,9 \%$, $81,8 \%$ y $79,6 \%$ respectivamente. Un $54 \%$ de los egresados considera se encuentra en un nivel 3 respecto a la competencia "resuelvo problemas con rigor científico". Las competencias reportadas en el nivel 2, fueron: comunicación en inglés en situaciones y temáticas cotidianas $(26,3 \%)$, comunicación escrita $(7,3 \%)$, uso de tecnologías de información y comunicación $(7,3 \%)$. Ningún egresado reportó encontrarse en el nivel 1. Conclusiones. En la dimensión trabajo grupal y académico, la competencia de tecnologías de información y comunicación, y dominio de idioma no lograron ser auto percibidas como logradas en la mayoría de egresados a un nivel 4. Igualmente, en la dimensión de relaciones interpersonales, la competencia de comunicación tanto oral como escrita no fue percibida como lograda en el máximo nivel.
\end{abstract}

Palabras clave: Competencia profesional; Odontología; Educación superior (fuente: DeCS BIREME).

\begin{abstract}
Objective. Evaluate the self perception of dentistry graduates regarding the achievement of generic competences at the end of professional training. Methods. Quantitative approach, descriptive design, cross-sectional study. It was applied a structured survey comprised of three dimensions to 137 graduates 2016-2017 period. Four levels of achievement of competence were established (N1, N2, N3, and N4). Results. The commitment to personal and professional development; the commitment and responsibility with
\end{abstract}

\section{Artículo Original}

Flor Pachas-Barrionuevo ${ }^{1, a}$, Lola Sueng-Navarrete ${ }^{1, b}$ Jorge Vásquez-Alva ${ }^{1, c}$, Wilfredo Mormontoy-Laurel ${ }^{2, d}$

${ }^{1}$ Universidad Peruana Cayetano Heredia, Facultad de Estomatología. Lima, Perú.

${ }^{2}$ Universidad Peruana Cayetano Heredia, Facultad de Ciencias y Filosofía. Lima, Perú.

${ }^{a}$ Magíster en Estomatología.

b Magíster en Educación con mención en Docencia e Investigación en Educación Superior.

c Magíster en Administración en Salud.

d Magíster en Salud Pública

\section{Correspondencia:}

Flor de María Pachas Barrionuevo

Correo electrónico: flor.pachas@upch.pe

Av. Perú 1393, San Martín de Porres, Lima, Perú.

\section{Coautores:}

Lola Isabel Sueng Navarrete

lola.sueng@upch.pe

Jorge Enrique Vásquez Alva

jorge.vasquez@upch.pe

Wilfredo Mormontoy Laure

wilfredo.mormontoy@upch.pe

\section{Editor:}

Yuri Castro Rodríguez

Universidad Nacional Mayor de San Marcos, Perú.

Conflicto de intereses: los autores declaran no tener conflictos de interés.

Fuente de financiamiento: autofinanciado.

Recibido: 26/04/19

Aceptado: 28/08/19

Publicado: 27/11/19

(C) Los autores. Este artículo es publicado por la revista Odontología Sanmarquina de la Facultad de Odontología, Universidad Nacional Mayor de San Marcos. Este es un artículo de acceso abierto, distribuido bajo los términos de la licencia Creative Commons Atribucion - No Comercia_Compartir Igual 4.0 Internacional. (http://creativecommons.org/licenses/by-nc-sa/4.0/) que permite el uso no comercial, distribución y reproducción en cualquier medio, siempre que la obra original sea debidamente citada. 
the social environment and the environment, as well as the demonstration of behavior and ethical commitment were the most reported competences in level 4 , with values of $83.9 \%, 81.8 \%$ and $79.6 \%$ respectively. $54 \%$ of the graduates considered to be in level 3 with according to the competence of solving problems with scientific rigor. The competences reported in level 2 were: communication in English in everyday situations and topics $(26.3 \%)$, written communication $(7.3 \%)$, use of information and communication technologies (7.3\%). No graduates reported finding themselves in level 1. Conclusions. In the group and academic work dimension, the competence of TIC' and language proficiency failed to be self-perceived as achieved in the majority of graduates at a level 4 . Similarly, in the interpersonal relations dimension, the competence of both oral and written communication was not perceived as achieved at the highest level.

Keywords: Periodontitis; Cytokines; Inflammation (source: MeSH NLM).

\section{Introducción}

La adquisición de las competencias, tanto genéricas como específicas, es un objetivo central en el proceso formativo. La enseñanza de las competencias en educación superior, debe cumplir un conjunto de características como relevancia con el entorno profesional, transferibilidad al estudiante y posibilidad de ser evaluada objetivamente ${ }^{1}$. Diversas investigaciones señalan una brecha entre la formación académica universitaria y el mundo laboral, originado por la deficiencia en cuanto a habilidades de trabajar en equipo, relaciones interpersonales, adaptación al cambio, control de emociones, entre otros ${ }^{2}$. Es así que las competencias genéricas corresponden a un saber comportamental, que son atributos personales de carácter cognitivo, social, actitudinal o valorativo, que complementa el comportamiento profesional ${ }^{3,4}$. De acuerdo al Proyecto Tunning, éstas se expresan como competencias instrumentales, interpersonales y sistémicas ${ }^{5}$.

Bennet et al. ${ }^{6}$ y Marzo et al. ${ }^{7}$ proponen clasificaciones similares de las competencias genéricas como la capacidad de adaptación, trabajar en equipo, habilidad de comunicación oral y escrita, manejo de la informática e idioma, habilidad para el aprendizaje, creatividad o trabajar en forma independiente y la competencia participativa, entre otros.

Asimismo, Villa y Poblete ${ }^{8}$ mencionan que las competencias genéricas presentan tres características, son funcionales, complejas y multidimensionales que contribuyen desarrollar un ciudadano integral, capaz de reflexionar y analizar los problemas de la sociedad, para resolverlos con un actuar ético, habilidades de trabajo en equipo, habilidades sociales, de comunicación y de interacción con personal de diferentes disciplinas. Ortiz et al. ${ }^{9}$ y Cárdenas et al. ${ }^{10}$ mencionan que el pensamiento crítico-reflexivo es un proceso de razonamiento complejo, intencional y autodirigido, donde se analiza la estructura y firmeza de las opiniones o afirmaciones, evaluando las ideas, juicios o acciones propias o ajenas, antes de la toma de decisiones o para resolver problemas. En el campo de la salud, se requiere razonamiento clínico, basado en la evidencia, para evaluar información, interpretar datos y explorar argumentos mediante mecanismo intelectuales de discriminación, precisión y juicio. Asimismo, Ortiz et al. ${ }^{9}$ en cuanto a responsabi- lidad social refieren que cuenta con tres elementos, una responsabilidad ante sí mismo, responsabilidad ante los cercanos y responsabilidad ante el entorno.

López ${ }^{11}$ menciona que la competencia cultural en salud alude a la habilidad, el conocimiento, la sensibilidad de los prestadores de salud para entender y responder en forma eficaz, efectiva, congruente y vinculada de manera afectiva a la cultura de las personas, familias y comunidades.

Por otra parte, la competencia de comunicación en el campo profesional y la vida diaria implica que un sujeto tenga conocimiento del lenguaje, de destrezas prácticas de tecnologías e información y la actitud y disposición para entablar una comunicación con otros ${ }^{12}$, entendiéndose como lenguaje al intermediario de la acción mental que permite que el estudiante aprenda, reflexione y exprese en el diálogo para el proceso de construcción del conocimiento ${ }^{13}$.

Gallego y Mora ${ }^{14}$ en un estudio de las competencias hallaron que menos de la mitad de empleadores considera que los odontólogos deben poseer la competencia de trabajo en equipo, habilidades comerciales, entre otros. Las menos valoradas fueron las competencias de comunicación, principios éticos, humanísticos y sociales. Chiarria et al. ${ }^{15}$ al respecto de esta situación consideran que los planes de estudios deben ser reevaluados por los responsables de la educación superior para el logro de los propósitos del programa académico y lo demandado por el mercado laboral.

En Perú, el Sistema Nacional de Evaluación, Acreditación y Certificación de la Calidad Educativa, exige a las universidades que el perfil de egreso de cada carrera oriente a la gestión del programa de estudio, el mismo que debe ser coherente con los propósitos, el currículo y dar respuesta a las expectativas de los grupos de interés y del entorno socioeconómico. Esto implica, que las carreras tienen la responsabilidad de evaluar el avance del logro de las competencias en el proceso de enseñanza-aprendizaje y evaluación ${ }^{16}$. En este sentido la presente investigación tuvo como objetivo determinar la autopercepción de los egresados de Odontología en cuanto al logro de las competencias genéricas al término de la formación profesional. 


\section{Métodos}

Enfoque cuantitativo, diseño descriptivo y transversal. La población de estudio perteneció a dos promociones de egresados de Odontología (Estomatología) de la Universidad Peruana Cayetano Heredia (UPCH), en Lima-Perú. Se utilizó la población de egresados de los años 2016 y 2017, cuyo criterio de inclusión fue la participación voluntaria y ser egresado que estudió los cinco años de la carrera en la institución universitaria. Se comunicó con los egresados después de la culminación de sus estudios, cuatro meses posteriores al egreso y se les preguntó acerca de los niveles de logro de las competencias genéricas (Tabla 1).

Tabla 1. Nivel de Logro de Competencia Genérica

\begin{tabular}{ll}
\hline Niveles & Definición \\
\hline Nivel 1 & $\begin{array}{l}\text { Competencia lograda en actividades elementales y necesi- } \\
\text { ta un apoyo total para demostrar el desenvolvimiento. }\end{array}$ \\
Nivel 2 & $\begin{array}{l}\text { Competencia lograda en actividades elementales, con } \\
\text { mínima autonomía y requiere un acompañamiento. }\end{array}$ \\
Nivel 3 & $\begin{array}{l}\text { Competencia lograda en la mayoría de actividades, se } \\
\text { requiere de una mínima orientación de un experto. }\end{array}$ \\
Nivel 4 & $\begin{array}{l}\text { Competencia lograda demostrada en diversas activi- } \\
\text { dades, en diferentes escenarios, con autonomía, y con } \\
\text { responsabilidad. }\end{array}$ \\
\hline
\end{tabular}

Las fases de validación del instrumento fueron cuatro. La primera fase se basó en la construcción teórica propuesta por Marzo et al. ${ }^{7}$ y González y González ${ }^{4}$. Desde el planteamiento de Marzo et al. ${ }^{7}$ las competencias genéricas las clasifica en tres dimensiones: social, personales y académicas, considerando que éstas son valoradas en las prácticas pre profesionales dentro de una empresa u organización. Esta propuesta surgió a inicios del siglo XXI, presentado en un Congreso de innovación educativa en España. Asimismo, González y González ${ }^{4}$ consideraron cuatro categorías: competencias relativas al aprendizaje, competencias relativas a las relaciones interpersonales y el trabajo grupal, competencias vinculadas a la autonomía y desarrollo personal y finalmente competencias relativas a los valores. Se mencionan que éstas son claves en un profesional, que unido a sus cualidades motivacionales y cognitivas produce un profesional eficiente y que sabe desenvolverse en cualquier contexto. A partir de ambas conceptualizaciones, se generó las tres dimensiones para este estudio: personal y autonomía (CG1, CG2, CG6, CG7, CG8), trabajo grupal y académica (CG3.1, CG3.2, CG3.3, CG4.4, CG4.5 y CG5) y relaciones interpersonales (CG4.1, CG4.2, CG 4.3). Los indicadores o ítems de cada dimensión, procedieron de las competencias genéricas declaradas en el modelo educativo de la institución universitaria. Esta agrupación se corroboró con el análisis factorial confirmatorio (Tablas 2 y 3 ).

La segunda fase consideró el juicio de expertos que estuvo conformado por cinco jueces, con experiencia profesional, académica y de investigación. De acuerdo a Aiken ${ }^{17}$, el valor de coeficiente de validez está de acuerdo al número de jueces o evaluadores (2 a 25 jueces)

Tabla 2. Análisis factorial de las competencias genéricas

\begin{tabular}{lcccc}
\hline \multicolumn{1}{c}{ Indicadores } & Dimensión $\mathbf{1}$ & Dimensión $\mathbf{2}$ & Dimensión $\mathbf{3}$ & Total \\
\hline CG1 & 0,7714 & 0,1437 & 0,1347 & 0,3662 \\
CG2 & 0,7538 & 0,2133 & 0,0170 & 0,3860 \\
CG3,1 & 0,5299 & 0,4352 & 0,0803 & 0,5234 \\
CG3,2 & 0,4781 & 0,5690 & 0,2447 & 0,3877 \\
CG3,3 & 0,4107 & 0,6236 & 0,2631 & 0,3733 \\
CG4,1 & 0,2321 & 0,7427 & 0,2793 \\
CG4,2 & 0,3391 & 0,5577 & 0,8115 & 0,2571 \\
CG4,3 & 0,1341 & 0,1819 & 0,7912 & 0,3046 \\
CG4,4 & 0,1907 & 0,6519 & 0,2242 & 0,5078 \\
CG4,5 & 0,1303 & 0,7134 & 0,2627 & 0,4118 \\
CG5 & 0,5771 & $-0,0166$ & 0,5063 \\
CG6 & 0,1011 & 0,0643 & 0,2928 & 0,3241 \\
CG7 & 0,4004 & 0,0660 & 0,3973 & 0,3139 \\
CG8 & 0,7655 & 0,2567 & 0,3172 & 0,2760 \\
\hline
\end{tabular}

Tabla 3. Matriz de la rotación de dimensiones

\begin{tabular}{lccc}
\hline & Dimensión 1 & Dimensión 2 & Dimensión 3 \\
\hline Dimensión 1 & 0,7123 & 0,5016 & 0,4909 \\
Dimensión 2 & $-0,6983$ & 0,4366 & 0,5672 \\
Dimensión 3 & $-0,0702$ & 0,7468 & $-0,6613$ \\
Las dimensiones quedan conformadas con los siguientes ítems: & \\
Dimensión 1 (personal y autonomía): CG1, CG2, CG6, CG7, CG8 & \\
Dimensión 2 (trabajo grupal y académica): CG3,1, CG3,2, CG3,3, CG4,4, CG4,5 y CG5 \\
Dimensión 3 (relaciones interpersonales): CG4,1, CG4,2, CG 4,3 \\
\hline
\end{tabular}


y al número de categorías. En este estudio se convocó a ocho jueces pero solo respondieron cinco y con ello se garantiza una validez de contenido estadísticamente significativo con un $p=0,021$. Al aplicar el método estadístico de $\mathrm{V}$ de Aiken con los cinco jueces se obtuvo un valor de 0,84 para validar el contenido. La tercera fase consideró la selección de una muestra piloto de 30 egresados con condiciones similares al grupo de estudio, una única evaluación se ejecutó para comprobar la comprensión de los ítems, aclaraciones y manejo del tiempo. La cuarta fase comprendió la medición de la fiabilidad del instrumento con el coeficiente alfa el cual alcanzó un valor de 0,83 . Los valores de los coeficientes por dimensión se reporta en la tabla 4. Se eligió este coeficiente porque los niveles de logro evaluados para cada competencia denotan una valoración ordinal. Un encuestador fue capacitado para la aplicación del instrumento, toma de contacto y recolección de datos de los egresados. Por correo electrónico, se remitió una encuesta estructurada, generada a partir del programa Google Forms, se midieron 14 ítems, correspondientes a las competencias genéricas declaradas en el perfil de egreso. Se solicitó a cada egresado respondiera acerca de la autopercepción del nivel de logro de cada competencia que poseían, clasificado en cuatro niveles. Se indicó que seleccione un nivel por cada pregunta. Las respuestas generaron una base de datos automatizada, que se declara en el Sistema Descentralizado de Información y Seguimiento a la Investigación (SIDISI) de la institución.

Tabla 4. Coeficiente alfa según dimensiones

\begin{tabular}{lc}
\hline Dimensión $\mathbf{1}$ (personal y autonomía) & \\
\hline Ítem & Alfa $(\alpha)$ \\
CG1 & 0,8502 \\
CG2 & 0,8586 \\
CG6 & 0,8422 \\
CG7 & 0,8416 \\
CG8 & 0,8286 \\
Valor del test & 0,8716 \\
\hline Dimensión 2 (trabajo grupal y académica) & \\
\hline Ítem & Alfa $(\alpha)$ \\
CG3,1 & 0,7506 \\
CG3,2 & 0,7875 \\
CG3,3 & 0,7575 \\
CG4,4 & 0,7510 \\
CG4,5 & 0,7148 \\
CG5 & 0,7081 \\
Valor del test & 0,7785 \\
\hline Dimensión 3 (relaciones interpersonales) & \\
\hline Ítem & Alfa $(\alpha)$ \\
CG4,1 & 0,7226 \\
CG4,2 & 0,7275 \\
CG 4,3 & 0,7888 \\
Valor del test & 0,8165 \\
\hline
\end{tabular}

La información final se procesó en el programa Excel, se calculó frecuencias absolutas y relativas por cada competencia de acuerdo al nivel de logro. Se aplicó la prueba
Chi-cuadrada con un nivel de confianza del 95\%, para comparar ambas promociones. Asimismo, se empleó el programa estadístico STATA versión 15, para el análisis factorial y los valores de alfa. El proyecto se registró en el SIDISI de la UPCH y fue aprobado por el Comité Institucional de Ética.

\section{Resultados}

De las 137 encuestas aplicadas a los egresados promociones 2016-2017, la competencia con mayor porcentaje de logro (Nivel 4) entre los egresados fue "Me comprometo con mi desarrollo personal y profesional" correspondiendo a un $83,8 \%$. En el nivel 3 la competencia más reportada fue "Resuelvo problemas con rigor científico" con 53,7\%. En el nivel 2 la competencia "Me comunico en inglés en situaciones y temáticas cotidianas", fue la que obtuvo el mayor porcentaje con $26,5 \%$. Ningún egresado autopercibió que sus competencias genéricas se encontraran en el nivel 1 (Figura).

Las competencias más reportadas en el nivel 4 correspondieron a: "Me comprometo con mi desarrollo personal y profesional", "Demuestro compromiso y responsabilidad con mi entorno social y medio ambiente", "Demuestro comportamiento y compromiso ético" y "Me desempeño eficazmente en distintos contextos socioculturales y profesionales" con valores $83,8 \%$, $82,4 \%, 79,4 \%$ y $73,5 \%$ respectivamente. La competencia de nivel 4 con menor porcentaje de reporte de logro correspondió a "Me comunico en inglés en situaciones y temáticas cotidianas" $(33,8 \%)$.

Las competencias más reportadas en nivel 3 correspondió a: "Resuelvo problemas con rigor científico" $(53,7 \%)$, seguido de "Utilizo tecnologías de la información y comunicación" (44,1\%). La competencia de nivel 3 con menor porcentaje de reporte de logro correspondió a: "Me comprometo con mi desarrollo personal y profesional" (14,0\%).

La competencia más reportada en nivel 2 correspondió a: "Me comunico en inglés y en situaciones y temáticas cotidianas" (26,5\%).

Más de la mitad de los egresados reportaron no alcanzar tres de los 14 items correspondientes a las competencias genéricas: "Utilizo tecnologías de la información y comunicación" (N4: 49,3\%; N3: 44,1\% N2: 6,6\%), "Me comunico en inglés en situaciones y temáticas cotidianas" (N4: 33,8\%; N3: 39,7\% y N2: 26,5\%) y "Resuelvo problemas con rigor científico (N4: 43,4\%; N3: $53,7 \%, \mathrm{~N} 2: 2,9 \%)$.

Para la promoción de egresados del año 2016, el 50,8\% consideró que la competencia "Me comunico en inglés en situaciones y temáticas cotidianas" fue lograda en un nivel 4. Todas las otras competencias oscilaron entre $57,6 \%$ a $89,8 \%$. Siete de los catorce indicadores (CG 1, CG 3.2, CG 4.1, CG 4.3, CG 6, CG 7 y CG 8) correspondientes a las competencias genéricas evaluadas acumuló más del $75 \%$ de egresados que consideran haber alcanzado un nivel 4. Para la promoción de egresados del año 2017, el 20,8\% consideró que la 
competencia "Me comunico en inglés en situaciones y temáticas cotidianas" fue lograda en un nivel 4. Todas las otras competencias oscilaron entre $32,5 \%$ a $79,2 \%$. Tres de los catorce indicadores (CG 1, CG 6 y CG 7) de las competencias genéricas acumularon más del 75\% de egresados que consideran haber alcanzado un nivel 4.

Al comparar los niveles de logro de las competencias entre las promociones 2016 y 2017 (Tabla 5), se halló diferencia significativa $(p<0,05)$ en nueve de ellos, correspondiendo a: CG $3.2(p=0,010)$, CG $3.3(p=$ $0,003)$, CG $4.1(p=0,000)$, CG $4.2(p=0,002)$, CG $4.3(p<0,001)$, CG $4.4(p=0,003)$, CG $4.5(p<0,001)$ CG $5(p=0,004)$, y CG $8(p=0,005)$.

\section{Discusión}

Las instituciones de educación superior cuando forman a las futuras generaciones, deben ser capaz de responder a las siguientes interrogantes: ¿Qué competencias formar y

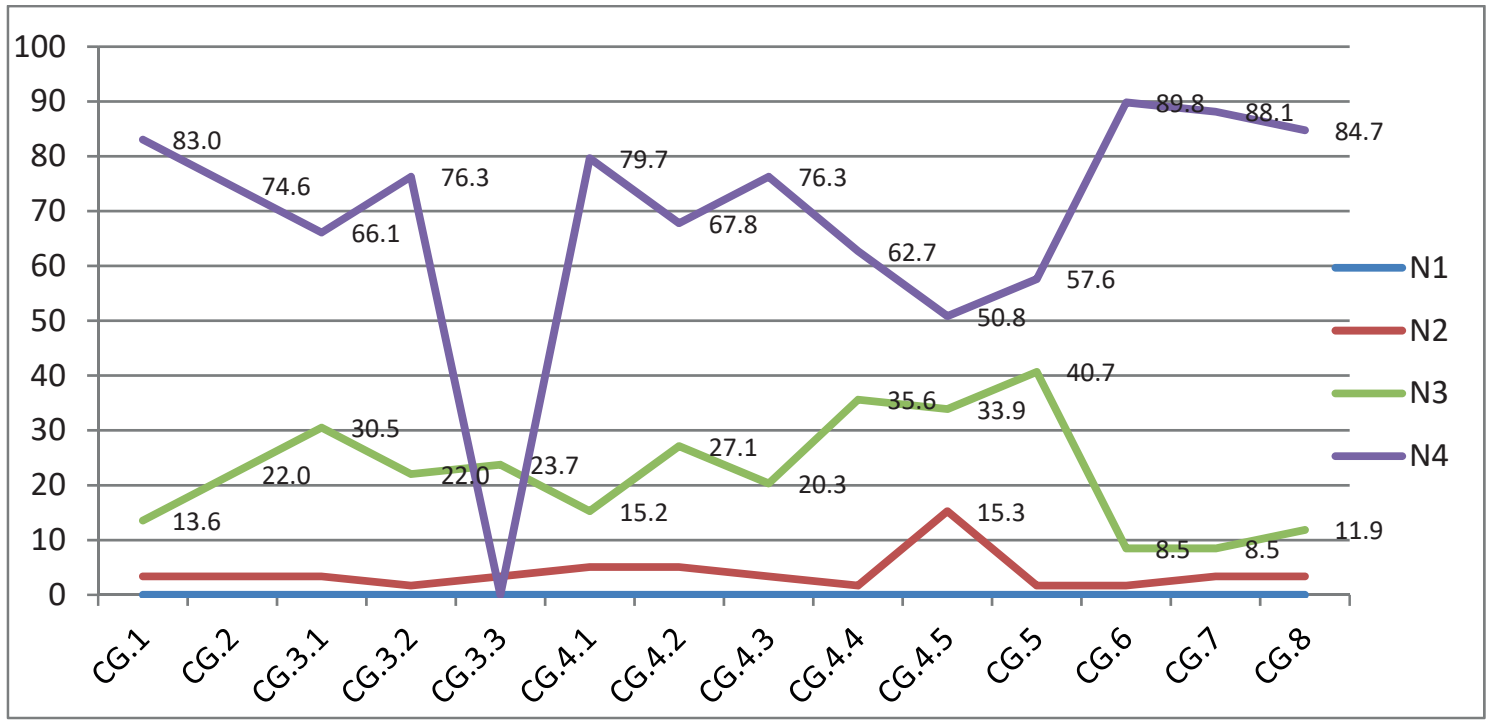

Figura. Distribución de los niveles de competencias genéricas en egresados de Odontología

Tabla 5. Competencias genéricas de egresados de Odontología, promociones 2016 y 2007

\begin{tabular}{|c|c|c|c|c|c|c|c|c|c|}
\hline \multirow{2}{*}{ Competencias genéricas (CG) } & \multicolumn{4}{|c|}{ Año 2016} & \multicolumn{4}{|c|}{ Año 2017} & \multirow[b]{2}{*}{$\mathrm{p}$} \\
\hline & N1 & N2 & N3 & N4 & N1 & N2 & N3 & N4 & \\
\hline CG 1, Demuestro comportamiento y compromiso ético, & $\begin{array}{c}0 \\
0 \%\end{array}$ & $\begin{array}{c}2 \\
3,4 \%\end{array}$ & $\begin{array}{c}8 \\
13,6 \%\end{array}$ & $\begin{array}{c}49 \\
83,0 \%\end{array}$ & $\begin{array}{c}0 \\
0 \%\end{array}$ & $\begin{array}{c}1 \\
1,3 \%\end{array}$ & $\begin{array}{c}17 \\
22,1 \%\end{array}$ & $\begin{array}{c}59 \\
76,6 \%\end{array}$ & 0,142 \\
\hline CG 2, Soy crítico, innovador y creativo, & $\begin{array}{c}0 \\
0 \%\end{array}$ & $\begin{array}{c}2 \\
3,4 \%\end{array}$ & $\begin{array}{c}13 \\
22,0 \%\end{array}$ & $\begin{array}{c}44 \\
74,6 \%\end{array}$ & $\begin{array}{c}0 \\
0 \%\end{array}$ & $\begin{array}{c}1 \\
1,3 \%\end{array}$ & $\begin{array}{c}23 \\
29,9 \%\end{array}$ & $\begin{array}{c}53 \\
68,8 \%\end{array}$ & 0,207 \\
\hline CG 3,1 Asumo responsabilidades compartidas, & $\begin{array}{c}0 \\
0 \%\end{array}$ & $\begin{array}{c}2 \\
3,4 \%\end{array}$ & $\begin{array}{c}18 \\
30,5 \%\end{array}$ & $\begin{array}{c}39 \\
66,1 \%\end{array}$ & $\begin{array}{c}0 \\
0 \%\end{array}$ & $\begin{array}{c}3 \\
3,9 \%\end{array}$ & $\begin{array}{c}27 \\
35,1 \%\end{array}$ & $\begin{array}{c}47 \\
61,0 \%\end{array}$ & 0,544 \\
\hline CG 3,2 Trabajo en forma cohesionada e integrada, & $\begin{array}{c}0 \\
0 \%\end{array}$ & $\begin{array}{c}1 \\
1,7 \%\end{array}$ & $\begin{array}{c}13 \\
22,0 \%\end{array}$ & $\begin{array}{c}45 \\
76,3 \%\end{array}$ & $\begin{array}{c}0 \\
0 \%\end{array}$ & $\begin{array}{c}4 \\
5,2 \%\end{array}$ & $\begin{array}{c}30 \\
39,0 \%\end{array}$ & $\begin{array}{c}42 \\
54,5 \%\end{array}$ & $0,010^{*}$ \\
\hline CG 3,3 Participo del trabajo interdisciplinario, & $\begin{array}{c}0 \\
0 \%\end{array}$ & $\begin{array}{c}2 \\
3,4 \%\end{array}$ & $\begin{array}{c}14 \\
23,7 \%\end{array}$ & $\begin{array}{c}43 \\
72,9 \%\end{array}$ & $\begin{array}{c}0 \\
0 \%\end{array}$ & $\begin{array}{c}7 \\
9,1 \%\end{array}$ & $\begin{array}{c}33 \\
42,9 \%\end{array}$ & $\begin{array}{c}36 \\
46,8 \%\end{array}$ & $0,003^{*}$ \\
\hline CG 4,1 Soy competente en la comunicación oral, & $\begin{array}{c}0 \\
0 \%\end{array}$ & $\begin{array}{c}3 \\
5,1 \%\end{array}$ & $\begin{array}{c}9 \\
15,2 \%\end{array}$ & $\begin{array}{c}47 \\
79,7 \%\end{array}$ & $\begin{array}{c}0 \\
0 \%\end{array}$ & $\begin{array}{c}5 \\
6,5 \%\end{array}$ & $\begin{array}{c}37 \\
48,1 \%\end{array}$ & $\begin{array}{c}34 \\
44,2 \%\end{array}$ & $<0,001^{*}$ \\
\hline CG 4,2 Soy competente en la comunicación escrita, & $\begin{array}{c}0 \\
0 \%\end{array}$ & $\begin{array}{c}3 \\
5,1 \%\end{array}$ & $\begin{array}{c}16 \\
27,1 \%\end{array}$ & $\begin{array}{c}40 \\
67,8 \%\end{array}$ & $\begin{array}{c}0 \\
0 \%\end{array}$ & $\begin{array}{c}7 \\
9,1 \%\end{array}$ & $\begin{array}{c}38 \\
49,4 \%\end{array}$ & $\begin{array}{c}32 \\
41,6 \%\end{array}$ & $0,002^{*}$ \\
\hline CG 4,3 Poseo comprensión lectora, & $\begin{array}{c}0 \\
0 \%\end{array}$ & $\begin{array}{c}2 \\
3,4 \%\end{array}$ & $\begin{array}{c}12 \\
20,3 \%\end{array}$ & $\begin{array}{c}45 \\
76,3 \%\end{array}$ & $\begin{array}{c}0 \\
0 \%\end{array}$ & $\begin{array}{c}4 \\
5,2 \%\end{array}$ & $\begin{array}{c}39 \\
50,6 \%\end{array}$ & $\begin{array}{c}33 \\
42,9 \%\end{array}$ & $<0,001^{*}$ \\
\hline $\begin{array}{l}\text { CG 4,4 Utilizo tecnologías de la información y comu- } \\
\text { nicación, }\end{array}$ & $\begin{array}{c}0 \\
0 \%\end{array}$ & $1,7 \%$ & $\begin{array}{c}21 \\
35,6 \%\end{array}$ & $\begin{array}{l}37 \\
62,7 \%\end{array}$ & $\begin{array}{c}0 \\
0 \%\end{array}$ & $\begin{array}{c}8 \\
10,4 \%\end{array}$ & $\begin{array}{c}38 \\
49,4 \%\end{array}$ & $\begin{array}{c}30 \\
39,0 \%\end{array}$ & $0,003^{*}$ \\
\hline $\begin{array}{l}\text { CG } 4,5 \text { Me comunico en inglés en situaciones y temáticas } \\
\text { cotidianas, }\end{array}$ & $\begin{array}{c}0 \\
0 \%\end{array}$ & $\begin{array}{c}9 \\
15,3 \%\end{array}$ & $\begin{array}{c}20 \\
33,9 \%\end{array}$ & $\begin{array}{c}30 \\
50,8 \%\end{array}$ & $\begin{array}{c}0 \\
0 \%\end{array}$ & $\begin{array}{c}27 \\
35,1 \%\end{array}$ & $\begin{array}{c}33 \\
42,9 \%\end{array}$ & $\begin{array}{c}16 \\
20,8 \%\end{array}$ & $<0,001^{*}$ \\
\hline CG 5 Resuelvo problemas con rigor científico, & $\begin{array}{c}0 \\
0 \%\end{array}$ & $1,7 \%$ & $\begin{array}{c}24 \\
40,6 \%\end{array}$ & $\begin{array}{c}34 \\
57,6 \%\end{array}$ & $\begin{array}{c}0 \\
0 \%\end{array}$ & $\begin{array}{l}3 \\
3,9 \%\end{array}$ & $\begin{array}{c}48 \\
62,3 \%\end{array}$ & $\begin{array}{c}25 \\
32,5 \%\end{array}$ & $0,004^{*}$ \\
\hline $\begin{array}{l}\text { CG } 6 \text { Me comprometo con mi desarrollo personal y } \\
\text { profesional, }\end{array}$ & $\begin{array}{c}0 \\
0 \%\end{array}$ & $\begin{array}{c}1 \\
1,7 \%\end{array}$ & $\begin{array}{c}5 \\
8,5 \%\end{array}$ & $\begin{array}{c}53 \\
89,8 \%\end{array}$ & $\begin{array}{c}0 \\
0 \%\end{array}$ & $\begin{array}{c}2 \\
2,6 \%\end{array}$ & $\begin{array}{c}14 \\
18,2 \%\end{array}$ & $\begin{array}{c}61 \\
79,2 \%\end{array}$ & 0,093 \\
\hline $\begin{array}{l}\text { CG } 7 \text { Demuestro compromiso y responsabilidad con mi } \\
\text { entorno social y el medio ambiente, }\end{array}$ & $\begin{array}{c}0 \\
0 \%\end{array}$ & $\begin{array}{c}2 \\
3,4 \%\end{array}$ & $\begin{array}{c}5 \\
8,5 \%\end{array}$ & $\begin{array}{c}52 \\
88,1 \%\end{array}$ & $\begin{array}{c}0 \\
0 \%\end{array}$ & $\begin{array}{c}2 \\
2,6 \%\end{array}$ & $\begin{array}{c}15 \\
19,5 \%\end{array}$ & $\begin{array}{c}60 \\
77,9 \%\end{array}$ & 0,072 \\
\hline $\begin{array}{l}\text { CG } 8 \text { Me desempeño eficazmente en distintos contextos } \\
\text { socio-culturales y profesionales, }\end{array}$ & $\begin{array}{c}0 \\
0 \%\end{array}$ & $\begin{array}{c}2 \\
3,4 \%\end{array}$ & $\begin{array}{c}7 \\
11,9 \%\end{array}$ & $\begin{array}{c}50 \\
84,7 \%\end{array}$ & $\begin{array}{c}0 \\
0 \%\end{array}$ & $\begin{array}{c}2 \\
2,6 \%\end{array}$ & $\begin{array}{c}25 \\
32,5 \%\end{array}$ & $\begin{array}{c}50 \\
64,9 \%\end{array}$ & $0,005^{*}$ \\
\hline
\end{tabular}

Nota: $* p<0,05$ 
por qué? ¿Hacia dónde orientar la formación de competencias? Para ello, se debe contar con un soporte tecnológico, promover un desarrollo y una formación académica de sus profesores y directivos, y asegurar metodologías que promuevan las competencias declaradas ${ }^{18}$.

Esta investigación evaluó ocho competencias genéricas, distribuidas en catorce indicadores pertenecientes a la carrera de Odontología. La competencia ética, en el presente estudio identificó que la mayoría de los egresados de Odontología percibieron haber logrado el máximo nivel, al término de su carrera. $\mathrm{Al}$ respecto, Couceiro ${ }^{1}$ afirma que en la formación de personal de salud, a nivel de educación superior, es importante identificar los conflictos éticos de la práctica clínica y generar respuestas lógicas y prudentes, para que el estudiante aprenda de forma significativa un procedimiento, una forma de actuación y una sensibilización frente a los valores involucrados en la acción clínica, en un marco de democracia. De acuerdo a Garzón y Zárate ${ }^{19}$ el aprendizaje de la bioética, implica considerar al paciente como una unidad biológica, psicológica, social y ética y que el aprendizaje basado en problemas es la mejor estrategia para promover un comportamiento y compromiso ético. Otros autores como González et al. ${ }^{4}$, Chiarra et al. ${ }^{15} \mathrm{y}$ Alonso ${ }^{20}$ mencionan que ocupa un nivel bajo en orden de importancia en algunas instituciones y alto en otras. Agrega Vélez et al. ${ }^{2}$ que está influenciado por el compromiso con la calidad. Gallego y Mora ${ }^{14}$ refieren que esta competencia es prioritaria en el mundo del trabajo.

En cuanto a creatividad, en este estudio, cerca de tres cuartas partes de egresados de Odontología percibieron que lograron el más alto nivel, al opinar "Sí soy crítico, innovador y creativo". En este sentido, Vélez et al. ${ }^{2}$ consideran que la creatividad para resolver situaciones continúa siendo el resultado más esperado en la formación universitaria, contrariamente lo reportado por Alonso ${ }^{20}$. $\mathrm{Al}$ respecto, Villa y Poblete ${ }^{8}$ mencionan que esta competencia permite enfrentar la barrera de lo tradicional, es desafiar lo establecido como norma y se caracteriza por ser flexible y ofrecer la capacidad de visualizar las cosas de diferente ángulo o punto de vista para llegar a una solución.

Por otro lado, Cárdenas et al. ${ }^{10}$ al evaluar el aprendizaje del pensamiento crítico y reflexivo encontraron que un poco más de la mitad de estudiantes de enfermería consideran que tienen capacidades de razonamiento con evidencia y menos de la mitad tienen la habilidad de elaboración de preguntas pertinentes. Asimismo, Alonso ${ }^{20}$ menciona que estudiantes universitarios de psicología lo que más valoran es el razonamiento crítico. Es probable que la gran mayoría de egresados del programa de Odontología percibieran el logro de esta competencia, porque en su formación estuvieron expuestos a diversas técnicas o estrategias en escenarios reales en el campo clínico o comunitario y tuvieron que poner en juego esta capacidad.

Por otro lado, la competencia de trabajo en equipo en el presente estudio se evaluó a través de tres indicadores relacionados a responsabilidades compartidas, trabajo en forma cohesionada y trabajo interdisciplinario, donde se halló que cerca de dos tercios de los egresados de Odontología, consideran haber logrado el máximo nivel. González y Acevedo ${ }^{21}$, Clemente y Escriba ${ }^{12}$ y Alonso ${ }^{20}$ mencionan que los estudiantes en ciencias de la salud, otorgan una alta valoración a las competencias de emprendimiento, trabajo en equipo interdisciplinario, la toma de decisiones y capacidad para asumir responsabilidades, destacando la importancia de su participación activa durante el proceso de aprendizaje. Contrariamente, González y González ${ }^{4}$ otorgan una baja valoración. Es probable que en el presente estudio, la cantidad de horas en el plan de la carrera de Odontología sea limitada. En este sentido Villardón ${ }^{22}$ refiere que es necesario que se establezcan metas grupales, se produzca la habilidad de relación interpersonal, de organizar tareas, de resolver conflictos y de reflexión en equipo.

Por otra parte, la comunicación escrita y oral en el presente estudio, a opinión de los egresados de Odontología, fue lograda en su máximo nivel por un poco más de la mitad, situación preocupante por ser parte de las competencias básicas que adquiere un individuo desde la educación regular ${ }^{23}$. Al respecto, Corominas ${ }^{3}$ encontró una baja valoración en esta competencia en estudiantes de los últimos años de una carrera administrativa, contrariamente a lo hallado por Gonzáles y Gonzáles ${ }^{4}$ y Alonso ${ }^{20}$.

Una explicación de esta situación es analizada por Gallar ${ }^{13}$ quien menciona que existen deficiencias en la expresión oral y escrita de los estudiantes universitarios, debido a que no comprenden lo que leen, no sistematizan la información, omiten reglas gramaticales, los textos escritos no guardan coherencia y transcriben párrafos, sin articularlos a sus propias producciones escritas. En cuanto a la comunicación oral, existe dificultad de transmitir sus ideas, poca fluidez en la argumentación y defensa. Asimismo, se detecta poca capacidad de escucha e intolerancia frente a los argumentos de sus compañeros y pobre reflexión analítica.

Agrega Bustos et al. ${ }^{24}$ que es clave la comprensión lectora en un individuo porque este es un proceso que exige tres actividades: la obtención del significado, la incorporación de este significado en la memoria y la construcción de la inferencia, indispensable para una completa comprensión. De acuerdo a los datos del Programa de Evaluación Internacional de Estudiantes, la comprensión lectora está en proceso de desarrollo en la educación regular de los peruanos ${ }^{25}$.

En cuanto a dominio de un segundo idioma, el presente estudio encontró que solo un tercio de egresados de Odontología percibe haber alcanzado el máximo nivel de logro. Similar situación reportaron González y González ${ }^{4}$, Chiarra et al. ${ }^{15}$ y Clemente y Escriba ${ }^{12}$. El dominio de tres niveles es señalado por Villa y Poblete ${ }^{8}$. La problemática hallada se debería al limitado número de horas que la curricula otorga tanto a nivel básico y superior y al poco desarrollo de habilidades de comprender un idioma extranjero. En Perú, desde el año 2014 con la nueva ley universitaria $\mathrm{N}^{\circ} 30220{ }^{26}$ establecido 
por el Ministerio de Educación, exige que para la obtención del grado de bachiller, el egresado debe dominar un idioma extranjero.

De la misma manera, en relación al uso de tecnologías de información y comunicación (TIC) se tuvo como hallazgo que menos de la mitad de egresados de odontología refirió que alcanzó el máximo nivel de logro. En este sentido, Valencia et al. ${ }^{27}$ indica que las TIC en la educación favorecen los procesos de enseñanza y aprendizaje y orientan a la construcción de aprendizajes significativos. Por lo cual debe aprovecharse de sus características, tales como: el almacenamiento y transmisión de información; el dinamismo y el formalismo; la hipermedia y la multimedia; la interactividad y la conectividad, que permite el trabajo en red, trabajo grupal y colaborativo, tanto para los docentes como para los estudiantes. Humanante et al. ${ }^{28}$ encontraron que gran parte de los estudiantes de ciencias de la salud mencionan que no son muy competentes. Por otro lado, Velez et al. ${ }^{2}$ señalan que la habilidad en uso de TIC es una competencia que influye a corto plazo en la práctica profesional. Toda institución de educación superior debe adaptar los procesos de enseńanza aprendizaje apoyados por TIC, en los cuales los docentes planifiquen, regulen y orienten las actividades académicas mediante plataformas Moodle u otras tecnologías ${ }^{29}$. La brecha en los hallazgos de este estudio, se podrían deber a que muchos docentes utilizan estrategias de enseñanza aprendizaje tradicionales y sincrónicas, y emplean de manera limitada las bondades de las TIC y no aprovechan las potencialidades de la tecnología, a través de foros, portafolios virtuales, mapas conceptuales virtuales, y uso de software estadísticos, incluso utilización de los sistemas de evaluación, entre otros.

De manera similar, la competencia de resolución de problemas es una capacidad esencial en toda carrera. Villa y Poblete ${ }^{8}$ mencionan que un primer paso es identificar el problema, el cual supone la búsqueda de una solución basado en la lógica. Un problema puede tener múltiples rutas de solución y conlleva a seleccionar la mejor ruta. En este estudio se encontró que menos de la mitad de los egresados consideró haber alcanzado el máximo nivel de logro y la mayoría opinó que aún necesita el apoyo de un experto. $\mathrm{Al}$ respecto, Clemente y Escriba ${ }^{12}$, Alonso ${ }^{20}$ y Martínez ${ }^{30}$ hallaron que la resolución de problemas y la toma decisiones son las más valoradas por los estudiantes. Vélez et al. ${ }^{2}$ mencionan que a opinión de expertos y empresarios estas son las más exigidas en el campo laboral. La brecha en la apreciación del logro de esta competencia "Resuelvo problemas con rigor científico" se explicaría a que probablemente el término "rigor científico" contenido en el reactivo los egresados lo vincularon solo a los trabajos de investigación científica y no a la resolución de problemas de manera integral en el campo clínico o social.

En cuanto a la competencia de desarrollo personal y profesional, este estudio encontró que más de cuatro quintos de egresados de odontología alcanzaron el máximo logro, al expresar: "Me comprometo con mi desarrollo personal y profesional". De acuerdo a Ortiz et al. ${ }^{9}$ la responsabilidad ante sí mismo, implica un compromiso que asume el propio individuo con sus metas personales. Solanes et al. ${ }^{31}$ mencionan que entre las competencias más valoradas está la capacidad de aprender, proceso activo donde se establece metas personales y se planifica el logro de las mismas. Además, Alonso ${ }^{20}$, Corominas ${ }^{3} \mathrm{y}$ Martínez y Riopérez ${ }^{32}$ encontraron el desarrollar y mantenerse actualizado, dominio de las destrezas y conocimiento de la profesión, responsabilidad, y aprendizaje a lo largo de la vida. Villardón ${ }^{22}$ señala que es necesario el uso de recursos para el aprendizaje, tipo experiencial y basado en la investigación.

Entre las competencias vinculadas al ámbito personal, esta investigación encontró que más de cuatro quintos de los egresados expresaron haber alcanzado el máximo nivel de logro, al expresar "Demuestro compromiso y responsabilidad con mi entorno social y medio ambiente". Ortiz et al. ${ }^{9}$ mencionan que se alcanza una responsabilidad colectiva cuando nuestro actuar incide en el entorno que nos rodea.

Asimismo, la competencia expresada en "Me desempeño eficazmente en distintos contextos socioculturales y profesionales" estuvo entre las competencias genéricas con mayor nivel de logro. Vélez et al. ${ }^{2}$ mencionan que la valoración y respeto por la diversidad y multiculturalidad son las competencias de mayor influencia a corto plazo en la práctica profesional, a pesar de ello, no se da prioridad. Chiarra et al. ${ }^{15}$ señalan que las competencias vinculadas a la diversidad y multiculturalidad, obtienen altos puntajes en estudiantes de diversas carreras de pregrado. De Oliveira et al. ${ }^{33}$ y Arias y López 34 definen a la competencia cultural como una combinación de conocimientos, actitudes y habilidades necesarias para que los profesionales de salud sean hábiles en interactuar eficazmente con poblaciones, cultural y étnicamente, diversas. Esto significaría que los egresados de Odontología, cuando fueron estudiantes de pregrado probablemente estuvieron expuestos a un contacto con personas de diversas culturas.

Desde la perspectiva de los autores y en base a la revisión de la literatura, las competencias genéricas parten desde el diseño del currículo, tomando en cuenta las necesidades del entorno. Este a su vez, depende de cómo es estructurado el plan de estudio y los recursos disponibles desde lo humano a lo tecnológico. El propio docente debe ser un modelo referencial de dominio tanto de las competencias genéricas y específicas para promover y trasferir estas competencias. Asimismo, la institución debe identificar con precisión que competencias genéricas espera lograr, sus indicadores y cómo evaluarlas a lo largo de la formación integral. Paralelamente, existe necesidad de incorporar estrategias en la formación que ayuden a promover las competencias genéricas, tales como Aprendizaje Basado en Problemas, Aprendizaje Basado en Proyectos, Aprendizaje Colaborativo, Estudios de Casos, Debates, Foros, otros. 
Se asume que entre las fortalezas de los planes de estudios en carreras como Odontología u Estomatología, en el país, es que brindan una formación integral con áreas de estudios generales, de estudios específicos y de especialidad, y tiene una duración mínima de cinco años, de acuerdo a la Ley Universitaria 30220. Sin embargo, el valor del crédito solo hace referencia a las actividades presenciales y no consideran las horas de estudio fuera del ámbito universitario.

En particular, como es el caso de Odontología, de modalidad presencial con alta cantidad de horas para desempeño clínico en los planes de estudio, la evaluación por ejemplo, suele estar centrada en lo procedimental, es decir lo técnico, descuidando probablemente el evaluar las competencias genéricas con descriptores precisos relacionados a la comunicación (oral, escrita, comprensión lectora, uso TIC), resolución de problemas, juicio crítico, toma de decisiones, entre otros. La poca familiarización del diseño y empleo de rúbricas o lista de cotejo a cargo de los docentes, hace que el estudiante no reconozca que se espera que él logre en las competencias y cómo va progresando a través del tiempo en el logro de las mismas. En general, toda la formación integral debe ser medida y evaluada con herramientas que ayuden a demostrar que realmente se alcanza los propósitos declarados, por el modelo o concepción educativa de la institución universitaria.

Finalmente, de las tres dimensiones evaluadas se identificó que la dimensión relaciones interpersonales, es decir la competencia de comunicación oral, escrita y la comprensión lectora es la más débil. Por tanto, deben tener un seguimiento permanente y ser evaluado a lo largo de la formación para alcanzar los niveles óptimos. Asimismo, las diferencias encontradas entre los niveles de logro de competencia se explicarían porque cada persona posee un conjunto de conocimientos, capacidades, actitudes, valores y una experiencia única y personal, además de una velocidad innata de aprendizaje. De acuerdo a Villardón ${ }^{22}$ existe una serie de barreras en la organización educativa, tales como: la dificultad de comprender que la finalidad esencial de la enseñanza es ayudar a construir competencias, dificultad de comprender que la transferencia de lo aprendido no es inmediata y requiere ser asociado a diversos escenarios, el docente no asume la responsabilidad de motivación del estudiante en el aprendizaje y la creencia que la teoría debe preceder a la práctica.

Para una mayor comprensión y profundización acerca de las competencias genéricas sería importante que se realicen más estudios, incorporando metodología cualitativa, se efectúe un seguimiento a lo largo de la formación para reconocer el avance del logro de las competencias. A partir de este estudio se concluye que la mayoría de las competencias previstas por la carrera se lograron alcanzar, pero en diversos niveles de acuerdo a la autopercepción del egresado. Sin embargo, las competencias genéricas de comunicación, manejo de tecnología, información y comunicación, y dominio de otro idioma son percibidas aún en proceso de logro. La carrera debe generar mecanismos de mejora para alcanzar las competencias declaradas en el perfil de egreso.

\section{Referencias bibliográficas}

1. Couceiro A. Enseñanza de la bioética y planes de estudio basado en competencias. Educ Med. 2008;11(2):69-76.

2. Vélez AR, Delgado LD, Sánchez WC. Análisis prospectivo de las competencias genéricas Tuning-Alfa en la ciudad de Medellín al 2032. Ago Usb. 2018;18(1):130151.

3. Corominas E. Competencias genéricas en la formación universitaria. Rev Educ. 2001;325:299-321.

4. González V, González RM. Competencias genéricas y formación profesional: un análisis desde la docencia universitaria. Rev Iberoam Educ. 2008;47:185-209.

5. González J, Wagennar R, Beneitone P. Tuning-America Latina: Un proyecto de las universidades. Rev Iberoam Educ. 2004;35:151-164.

6. Bennet N, Dunne E, Carré C. Patterns of core and generic skills provision in higher education. Higher Educ. 1999;37:71-93.

7. Marzo M, Pedraja M, Rivera P. Las competencias profesionales demandadas por las empresas: el caso de los ingenieros. Rev Educ. 2006;34:643-661.

8. Villa A, Poblete M. Aprendizaje basado en competencias: Una propuesta para la evaluación de las competencias genéricas. Bilboa: Ediciones Mensajero; 2007.

9. Ortiz J, Fonseca G, Cantín M, Huitzil E, Lucena J. Competencias genéricas para la educación en odontología forense: pensamiento crítico y responsabilidad social. Int J Odontostomat. 2015;9(2):263-272.

10. Cárdenas L, Martínez B, Arana B, Monroy A. Desarrollo del pensamiento reflexivo y crítico en estudiantes de enfermería: evidencia de una universidad pública mexicana. Rev Urug Enferm. 2017;12(1):1-19.

11. López L. La competencia cultural una clave para mejores resultados en salud. Med UNAB. 2016;18(3):163-165.

12. Clemente J, Escribá C. Análisis de la percepción de las competencias genéricas adquiridas en la universidad. Rev Educ. 2013;362:535-561.

13. Gallar M. Trivium: una necesidad urgente y una responsabilidad compartida entre docentes y discentes. XIV Jornadas de Redes de investigación en docencia universitaria. Investigación, innovación y enseñanza universitaria: enfoques pluridisciplinares [Internet]. España: 2016. [Citado13 mar 2019]. Disponible en: http://rua.ua.es/ dspace/handle/10045/57093

14. Gallego C, Mora A. Competencias requeridas para la formación de odontólogos desde la perspectiva de los empleadores. Rev Colomb Invest Odontol. 2013;4(12):1-4.

15. Chiarria V, Sarsosa K, Ortíz F. Propiedades psicométricas de un instrumento de las competencias genéricas del egresado de una universidad privada de Cali, Colombia. Rev Divers Perspect Psicol. 2012;8(1):101-121.

16. SINEACE. Modelo de acreditación para programas de estudios de educación superior universitaria [Internet]. Perú: 2017 [citado 01 mar 2019]. Disponible en: https://www.sineace.gob.pe/ 
17. Aiken L. Three Coefficients for Analyzing the Reliability and Validity of Ratings. Educ Psychol Meas. 1985;45(1):131-142.

18. Rivera N. Las capacidades humanas desde la perspectiva personológica del profesional competente. Educ Med Sup. 2016;30(4):390-396.

19. Garzón F, Zárate B. El aprendizaje de la bioética basado en problemas (ABBP): Un nuevo enfoque pedagógico. Act Bioeth. 2015;21(1):19-28.

20. Alonso P. La valoración de la importancia de las competencias transversales: comparación de su percepción al inicio y final de curso en alumnos de psicología. Rev Invest Educ. 2010;28(1):119-140.

21. González A, Acevedo C. Percepción de estudiantes de la salud acerca de la implementación de las macrocompetencias genéricas. Educ Med Sup. 2016;30(4):349-360.

22. Villardón L. Competencias genéricas en educación superior. Madrid: NARCEA, S.A; 2015.

23. Ministerio de Educación. Currículo Nacional de la Educación Básica [Internet]. Perú: 2016. [citado 30 mar 2019]. Disponible en: http://www.minedu.gob.pe/curriculo/pdf/curriculo-nacional-de-la-educacion-basica.pdf

24. Bustos L, Salazar L, Moreno M. Estrategias metacognitivas y comprensión lectora en universitarios miembros de comunidades indígenas. Rev Col Reh. 2013;12:32-38.

25. Ministerio de Educación. Marco de evaluación de la competencia lectora de PISA [Internet]. Perú: 2018. [citado 30 mar 2019]. Disponible en http://umc.minedu.gob.pe/wp-content/uploads/2017/11/marco-fundamentacion-pisa.pdf

26. Ministerio de educación. Ley Universitaria $N^{\circ} 30220$ [Internet]. Perú: 2014. [citado 30 mar 2019]. Disponible en https:/www.sunedu.gob.pe/wp-content/ uploads/2017/04/Ley-universitaria-30220.pdf
27. Valencia T, Cerna A, Ochoa S, Caicedo A, Montes J, Chávez J. Competencias y estándares TIC desde la dimensión pedagógica: Una perspectiva desde los niveles de apropiación de las TIC en la práctica educativa docente. Cali: Pontificia Universidad Javeriana; 2016.

28. Humanante P, Solís M, Fernández J, Silva J. Las competencias TIC de los estudiantes que ingresan a la universidad: una experiencia en la facultad de ciencias de la salud de una universidad latinoamericana. Educ Med. [Internet]. 2018. [citado 30 mar 2019]. Disponible en : https://doi.org/10.1016/j.edumed.2018.02.002

29. Vilanova G. Modelos de interacción en ambientes virtuales de aprendizaje en la educación superior. Sist Cib Inform. 2016;13(1):77-83.

30. Martínez H. El enfoque por competencias desde la perspectiva del desarrollo humano: Aspectos básicos y diseño curricular. Av Psicol. 2013;21(1):9-22.

31. Solanes A, Núñez R, Rodríguez J. Elaboración de un cuestionario para la educación de competencias genéricas en estudiantes universitarios. Apuntes Psicol. 2008;26(1):35-49.

32. Martínez C, Riopérez N. Programa de desarrollo de competencias para el aprendizaje a lo a largo de la vida para estudiantes de educación superior. Pedagogía Social. Rev Interuniv [Internet]. 2013 [citado 30 mar 2019]; 22: 137-151. Disponible en https://www.redalyc.org/ html/1350/135031394010/

33. De Oliveira R, Damásio C, Lira L, Monteiro A, Lucía V, Dantas G. Incorporando a Competência Cultural para Atençấo à Saúde Materna em População Quilombola na Educação das Profissóes da Saúde. Rev Bras Educ Med [Internet]. 2018 [citado $30 \mathrm{mar}$ 2019];42(2):100-109. Disponible en http://dx.doi. org/10.1590/1981-52712015v42n2rb20170086

34. Arias S, López 1. Culture brokerage as a form of caring. Invest Educ Enferm. 2013;31(3):414-420. 
